\title{
LIFE-THREATENING DERMATOSES IN NEONATES AND NEWBORNS
}

\author{
Valentina Broshtilova ${ }^{1}$, Dimitar Nikolov ${ }^{2}$, Filka Georgieva ${ }^{2}$, Jeni Dimitrova ${ }^{2}$, \\ Sonya Marina ${ }^{2}$ \\ ${ }^{1}$ Department of Dermatology and Venereology, Faculty of Medicine, \\ Medical University-Sofia \\ ${ }^{2}$ Department of Infectious diseases, Parasitology and Dermatovenereology, \\ Faculty of Medicine, Medical University of Varna
}

\begin{abstract}
INTRODUCTION: Life-threatening dermatoses in newborns are versatile and extremely difficult for verification. Infectious diseases, developmental defects and complicated genodermatoses are among the most important causes.

MATERIALS AND METHODS: Perinatal history, mother's general state and epidemiologic data are the most important factors for differentiation and proper investigation. Clinical presentation and laboratory findings further support the proper management selection.
\end{abstract}

RESULTS: Treatment options always depend on proper diagnostic verification.

CONCLUSIONS: Herein, a thorough review of the most common life-threatening dermatoses in newborns is presented. A diagnostic algorithm and therapeutic clues are provided. Scr Sci Med. 2018;50(1):0nline First

Keywords: newborn, life-threatening dermatoses

According to the World Health Association the definition of a "newborn" is refer as a young offspring of human aged up to 12 months. A neonate belongs to the same age category; however, it is restricted to the first 28 days of life. This is an extremely vulnerable adaptation age, which requires specific care and very profound healthcare management. In

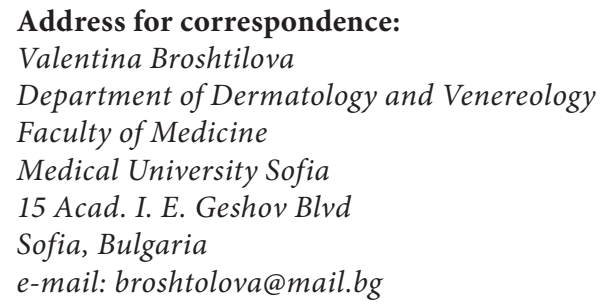

Received: August 4, 2017

Accepted: March 30, 2018 this age category, skin lesions are frequent and quite essential clues to more complicated hereditary syndromes. Fortunately, life-threatening skin conditions are rare. Among them, infectious diseases are the most frequent extrinsic dermatoses, while developmental defects (hamartomas) and genodermatoses represent the intrinsic dermatological entities (1).

\section{Intrinsic Dermatoses}

Developmental abnormalities encompass the spectrum of cranial or spinal dysraphism, encephaloceles, meningoceles, and combined cranial, suboccipital, or lumbosacrococcygeal midline defects (2). Obscure minor cutaneous signs can represent hyperpigmented macules, depressed lesions, polypoid nodules and subcutaneous indurations. They have to be early recognised by the localization and subjective neurological symptoms. Urgent ultrasonographic evaluation and magnetic resonance imaging has 
to be performed to facilitate the diagnosis and determine the proper neurosurgical management. Timely verification prevents further complications, such as severe life-threatening infections and pseudotumor cerebri.

Genodermatoses include severe hereditary keratinization disorders and epidermolysis bullosa. Those syndromes usually present at birth with collodion baby, a term that corresponds to the presence of shiny, tight film, resembling collodion skin cover (1). With time, most of the newborns develop ichthyosiform erythroderma or lamellar ichthyosis. Pathogenetically, collodion baby syndrome is due to the impaired barrier function of the skin (3). Therefore, hypothermia, dehydration, and metabolic dysfunction are the most common consequences. Superimposed bacterial infection is also a threat, and quite frequently, a cause for death. The increased absorption capacity leads to enhanced systemic toxicity, which makes the use of potent topical modalities very risky and dangerous. Neonatal intensive care and proper supportive management have to be organised. The mortality rate is still high, reaching up to $30 \%$ in countries with lack of specialised equipment and staff.

Harlequin foetus is the most severe neonatal dermatological condition. It is characterised by a thick plate-like covering of scales, which wrap the baby so firmly that distort surface features and restrict movements (4). Usually the neonate does not survive the first 24 hours. The rare cases, which obtain adequate intensive care and prompt introduction of retinoid therapy, usually develop a life-long, severe and painful ichthyosis. Parents of such neonates have to be very well informed for the bad longterm prognosis and psychological support has to be provided.

The neonates that present with extensive blisters and erosions at birth usually suffer simplex, junctional or dystrophic epidermolysis bullosa. Frictioninduced blistering is the hallmark of all types of keratinization genodermatoses. Usually, it is very difficult to differentiate clinically the subtype of epidermolysis. The most severe forms are Herlitz (junctional epidermolysis bullosa), Dowling-Meara (epidermolysis bullosa simplex), Hallopeau-Siemens (recessive dystrophic epidermolysis bullosa) (1). The affected newborns fail to thrive, become anaemic, and die of nutritional imbalance, despite food supplementation; septicaemia, or due to upper respiratory tract obstruction in the first months or years of life. Skin biopsy for routine analysis and ultrastructural diagnosis, together with bacterial smears, have to be processed. Genealogical analysis may be suggestive for the subtype, although many times it is not so informative. Careful handling and minimisation of minor traumas is essential, together with proper intensive care management.

Haemangiomas are the most common benign tumours in children. They are characterised by an initial phase of proliferation, followed by spontaneous regression. Life-threatening complications are rare, exceptionally seen in the first months of life.

The Kasabach-Merritt phenomenon is considered the most severe complication of tufted haemangioma or kaposiform haemangioendothelioma (5). The pathogenesis is related to thrombocytopaenia with subsequent consumption coagulopathy due to rapid enlargement of the tumour size. An alarming symptom is the fast tumour growth with the appearance of peripheral teleangiectasiae, petechiae, and ecchimoses. Platelet count drops and fibrinogen becomes undetectable. Histological analysis can be performed only in cases when malignancy is suspected, since some tumours can occur with transient thrombocytopaenia. No therapy is effective. The mortality rate is more than $30 \%$. The main therapeutic goal is the tumour size reduction, which secondarily improves the platelet count. Systemic or oral corticosteroids and vincristine are the first-line therapy. Weekly vincristine courses for a period of 4-12 weeks seem successful. Surgical and radiation therapy have also been discussed.

Airway haemangioma (6), which is usually subglottic, has to be suspected in cases with "beard" distribution of pulsatile subcutaneous tumour. Sometimes it can be complicated with a cutaneous segmental haemangioma that looks like an extended plaque in the preauricular region, chin, lower lip and anterior neck. Therefore, newborns with haemangiomas with such localization have to be strictly monitored for a croup-like cough, progressive biphasic stridor or other signs of obstruction. Endoscopic visualisation and embolisation are the proper therapeutic implication. 
Valentina Broshtilova, Dimitar Nikolov, Filka Georgieva et al.

Multifocal haemangiomatosis (7) defines an abrupt appearance of more than five vascular lesions, with a diameter of a few millimetres to centimetres in size, on skin at birth or shortly after. Sometimes extracutaneous localisations that can be life-threatening can evolve. Mortality is related to development of liver vascular shunts that lead to congestive heart failure. Gastrointestinal and intracranial haemorrhage with consumptive coagulopathy can also lead to a fatal outcome. All infants have to be completely screened for clinical symptoms of pulmonary obstruction, heart failure and gastrointestinal bleeding. Chest radiography, echocardiography, abdominal imaging, blood counts and coagulation studies are implicated in cases of clinical suspicion. The specific complications have to be managed accordingly by specialised teams.

\section{Extrinsic Dermatoses}

Neonatal infections can become life-threatening, especially in preterm infants with low weight and infants managed in intensive care units. Systemic infection cannot be diagnosed only by cutaneous symptoms, and requires analysis of the general appearance of the infant - type of crying, muscular tone, food intake. Hepatosplenomegalia, lymph node enlargement, neurological manifestations have also to be taken into account when verifying an infectious condition. The skin changes that are most commonly seen are bullous, pustular or vesicular lesions that are widely spread on the body surface.

Bacterial infections are represented by rapid distribution of erythematous patches in the second or third day of life. Staphyloccocus aureus or ß-haemolytic Streptococcus are the most aggressive agents. The most severe toxic staphylococcal disease is the 4-S syndrome (Staphylococcal scalded skin syndrome) (8). It is caused by diffusion of exfoliative toxin from an infected focus - omphalitis, conjunctivitis, etc. - which splits the spinous keratinocytes and causes complete shedding of the upper epidermal layer. Systemic syndromes occur when the toxin enters the blood circulation. A painful, erythematous, superficial blistering is seen around the affected focus, because neonates have some protective immunity transmitted from the mother. Very rarely, an extended exfoliative erythroderma can evolve, most often in neonates with impaired innate immunity.
Recovery is observed in most of the cases. Electrolyte disturbances and circulation failure can occur, increasing the risk of a lethal outcome. Identification of family members and healthcare givers, colonised with Staphylococcus aureus, is crucial for long-term prognosis and proper management.

Viral diseases with severe life-threatening complications that can occur in neonates and newborns are herpes and varicella infections. Neonatal herpes (9) may be a consequence of intrauterine infection. In such cases it appears in the first 2 days of life with extensive umbilicated vesicles and pustules that resorb with atrophic lesions and sometimes with aplasia of the skin scalp. Involvement of the nervous system is commonly seen, presented by microcephaly or hydrocephaly and ocular malformations. If the infection is acquired around delivery, it is usually characterised by circumscribed mucocutaneous lesions that appear in the first week of life. The best treatment is the immediate introduction of anti-viral modality intravenously for a period of 5-7 days.

Intrauterine exposure to varicella zoster virus leads to foetal varicella syndrome (with a fatal outcome) and neonatal varicella (10). The latter is represented by widespread vesicles and pustules, sometimes in association with respiratory distress, hepatitis or encephalitis. Prompt introduction of intravenous acyclovir $20 \mathrm{mg} / \mathrm{kg}$ every $8 \mathrm{~h}$ for a minimum of 8 days is crucial. Exceptional cases of herpes zoster can evolve in cases with neonatal exposure to the virus and insufficient count of maternal antibodies.

Fungal infections are observed in three main forms: congenital systemic, congenital non-systemic, and neonatal (napkin dermatitis) (11). Congenital forms follow intrauterine infection and are characterised by multiple papule-pustules on an erythematous base that affect face, neck, trunk, limbs, and oral cavity. All lesions resorb with tiny desquamation. Topical treatment is considered efficient in non-complicated cases. Systemic involvement develops almost always in infants with underlying immunodeficiency disorder and premature ones with low-birth weight. They have more extensive lesions with burn-like areas of denuded skin. Oral fluconazole is a first-line therapy in such cases (12). 


\section{CONCLUSION}

Life-threatening dermatoses in neonates and newborns are rare conditions that can be either caused by infectious agents or due to inherited genodermatosis, developmental defects, and hereditary neoplasms. Their proper management requires adequate diagnostic verification and multifunctional highly-specialised intensive care teams to eliminate the unfavourable prognosis and prevent a fatal outcome.

\section{REFERENCES}

1. Bodemer C. Main life-threatening dermatoses in neonatal period and in infancy. In: Revuz J, Roujeau JC, Kerdel F, Valeyrie-Allanore L, editors. Lifethreatening dermatoses and emergencies in dermatology. Berlin-Heidelberg: Springer-Verlag; 2009. p. 181-7.

2. Guggisberg D, Hadj-Rabia S, Viney C, Bodemer C, Brunelle F, Zerah M, et al. Skin markers of occult spinal dysraphism in children: a review of 54 cases. Arch Dermatol. 2004;140(9):1109-15. doi: 10.1001/ archderm.140.9.1109.

3. Sharma D, Gupta B, Shastri S, Pandita A, Pawar S. Collodion baby with TGM1 gene mutaton. Int Med Case Rep. 2015; 8:205-8. doi: 10.2147/IMCRJ. S91517.

4. Mithwani AA, Hashmi A, Shahnawaz S, Al Ghamdi Y. Harlequin ichthyosis: a case report of prolonged survival. BMJ Case Rep. 2014. doi: 10.1136/ bcr-2013-200884.

5. Hall GW. Kasabach-Merritt syndrome: pathogenesis and management. Br J Haematol. 2001; 112(4):851-62.

6. Orlow SJ, Isakoff MS, Blei F. Increased risk of symptomatic hemangiomas of the airway in association with cutaneous hemangiomas in a "beard" distribution. J Pediatr. 1997; 131(4): 643-6.

7. Holden KR, Alexander F. Diffuse neonatal hemangioma. Pediatrics. 1970; 46(3):411-21.

8. Dancer SJ, Simmons NA, Poston SM, Noble WC. Outbreak of staphylococcal scalded skin syndrome among neonates. J Infect. 1988; 16(1):87-103.

9. Hutto C, Arvin A, Jacobs R, Steele R, Stagno S, Lyrene $R$, et al. Intrauterine herpes simplex virus infection. J Pediatr. 1987; 110(1):129-50.

10. Enders G, Miller E, Craddock-Watson J, Bolley I, Ridehalgh M. Consequence of varicella and herpes zoster in pregnance. Prospective study of 1739 cases. Lancet. 1994; 343(8912): 1547-50.

11. Glassman BD, Muglia JJ. Widespread erythroderma and desquamation in a neonate. Congenital cutaneous candidiasis. Arch Dermatol. 1993; 129(7):899,902.

12. Dvorak AM, Gavaller B. Congenital systemic candidiasis. N Eng J Med. 1999; 274(10): 540-3. doi: 10.1056/NEJM196603102741003. 